\title{
Evaluation de la pollution générée par les lixiviats de la décharge publique de la ville de Fès
}

\author{
H. Chtioui', F. Khalil', S. Souabi' ${ }^{2}$ M.A. Aboulhassan \\ (I) Laboratoire de chimie appliquée, Faculté des sciences et techniques, BP-2202, Fès - Maroc \\ (2) Laboratoire de génie de l'eau et de l'environnement, Faculté des sciences et techniques, Mohammedia - Maroc
}

Pour toute correspondance : fouad.khalil@fst-usmba.ac.ma

\begin{abstract}
Résumé
Cette étude porte sur l'évaluation de la pollution de lixiviats produits par l'ancienne décharge de la ville de Fès. Le diagnostic de lixiviats a montré une forte pollution organique difficilement biodégradable qui évolue au cours du temps. En effet, la charge polluante produite par jour en DCO varie entre 20 et $216 \mathrm{~kg}$. En outre, la concentration en NTK varie autour de $4000 \mathrm{mg} / \mathrm{l}$, tandis la concentration en $\mathrm{NO}_{3}$ varie autour de $80 \mathrm{mg} / \mathrm{l}$. L'analyse des éléments métalliques a montré une importante concentration en chrome qui peut atteindre $9 \mathrm{mg} / \mathrm{l}$, tandis que la concentration en $\mathrm{Cu}, \mathrm{Zn}, \mathrm{Pb}$ et $\mathrm{Ni}$ dépasse les normes de rejet. Par ailleurs, les teneurs en éléments métalliques analysées dans les sédiments prélevés à partir du même point que les lixiviats sont importantes et varient d'un point de prélèvement à l'autre. Le chrome présente des teneurs qui dépassent I $250 \mu \mathrm{g} / \mathrm{g}$, tandis que les teneurs du $\mathrm{Pb}$ et du $\mathrm{Hg}$ dépassent respectivement 760 et 4,7 $\mu \mathrm{g} / \mathrm{g}$. Les concentrations maximales en $\mathrm{Cr}$ et en $\mathrm{Zn}$ analysées dans le compost sont respectivement de $480 \mathrm{mg} / \mathrm{g}$, et de I $320 \mathrm{mg} / \mathrm{g}$ tandis que le $\mathrm{Pb}$ présente $110 \mathrm{\mu g} / \mathrm{g}$. Ceci témoigne d'une pollution métallique des lixiviats provenant de la décharge brute qui reçoit toutes sortes de déchets, en particulier les déchets de tanneries, des margines, de textile, d'activités agroalimentaires...
\end{abstract}

Mots clés : décharge, lixiviat, pollution, métaux lourds

\section{Introduction}

Au Maroc, la production croissante des ordures ménagères $(\mathrm{OM})$ et des déchets industriels entraîne des problèmes de pollution critiques. La nature de plus en plus complexe et hétérogène de ces déchets implique des difficultés pour leur traitement et leur gestion. Une grande partie est mise en décharge, sans précautions, ce qui constitue une réelle et permanente menace pour l'environnement.

La décharge de la commune de Fès (route des Oudayas) reçoit plus de 800 tonnes par jour de déchets de toute nature. Cette quantité est largement supérieure à celles reçue par la décharge de Mohammedia (200 tonnes /jour) (Chtioui et al., 2005).
Les lixiviats produits en grande quantité dans des décharges où sont entreposés des déchets très humides et sans précaution vis-à-vis de la pluviométrie sont très chargés en polluants organiques issus en particulier des réactions de fermentation. Ces lixiviats peuvent contenir beaucoup de matières organiques (biodégradables, mais aussi réfractaires à la biodégradation) constituées dans la majeure partie par des substances humiques, mais aussi par de l'azote ammoniacal, des métaux lourds, des organochlorés et des sels inorganiques. Une partie de ces lixiviats s'infiltre dans le sol qui présente des fissures, ce qui peut engendrer une pollution des eaux souterraines. Une autre partie ruisselle et se déverse dans les eaux de l'oued Fès. Une grande partie du lixiviat finit généralement par s'infiltrer dans le sol ou par s'évaporer avant d'atteindre l'oued.

Les lixiviats sont une source de contamination des eaux de surface et des eaux souterraines s'ils ne sont pas correctement traités. L'étude de la qualité physico-chimique des eaux souterraines se trouvant près de la décharge de Casablanca a ainsi permis de déceler des teneurs élevées non seulement en éléments majeurs mais aussi en éléments métalliques émanant des lixiviats de la décharge. Ces derniers arrivent à la zone saturée et leur propagation est facilitée par la présence des failles qui affectent l'aquifère (Fekri et al., 2004).

Une étude d'impact et une quantification de la pollution du lixiviat s'avèrent donc nécessaires afin de pouvoir orienter correctement la collecte, le triage, la valorisation et le traitement des déchets solides. Le choix de la technique de traitement de lixiviat peut se révéler particulièrement difficile à cause de ses caractéristiques physico-chimiques variables dans le temps (Kerbachi et Belkacemi, 1994).

Cette étude a pour objectifs la quantification de la pollution des lixiviats de la décharge de la ville de Fès et de son évolution au cours du temps pour contribuer à la création d'une base de données relatives à la pollution des lixiviats des décharges publiques marocaines.

\section{Le site étudié}

La décharge publique de Fez (route des Oudayas) est utilisée depuis 1981. Elle arrive bientôt à saturation. C'est pourquoi les ordures de la ville de Fès sont envoyées à la nouvelle décharge contrôlée (route Sidi Harazem). En partie 
basse, le lixiviat ruisselle vers l'oued Fès avec un débit variant autour de $20 \mathrm{~m}^{3} /$ jour. Une grande partie du liquide s'infiltre dans le sol ou s'évapore avant d'atteindre le cours d'eau, sauf en période pluviale. Une couche de terre est parfois répandue sur les déchets pour éviter les incendies, surtout en été. De nombreuses personnes fréquentent cette décharge pour collecter des matériaux potentiellement recyclables qui sont source de revenus (flacons, métaux, os...). La décharge attire aussi des animaux qui y trouvent de la nourriture (vaches, chèvres, moutons, chiens, rats...).

Une classification des déchets mis en décharge à Fès révèle la présence de plusieurs catégories de déchets provenant d'ordures ménagères et de déchets professionnels. On trouve plusieurs types de matériaux tels que : papiers et cartons, plastiques, verre, métaux (boîtes de conserve et de peinture), os, textiles, margines, cuir... Cette diversité des déchets engendre des lixiviats riches en matières organiques et minérales, dont on peut craindre la grande toxicité.

\section{Méthodologie expérimentale}

Nous avons choisi les points de prélèvement de façon à ce que l'échantillonnage soit représentatif du milieu suivant sa situation par rapport aux sources de pollution. La figure I représente la répartition des différents points de prélèvements ( $\mathrm{PI}$ à $\mathrm{P9}$ ) choisis pour l'étude.

Les différents paramètres physico-chimiques des lixiviats (DCO, $\mathrm{DBO}_{5}, \mathrm{pH}$, oxygène dissous, $\mathrm{NTK}_{1} \mathrm{NO}_{3}, \mathrm{O}_{2}$ ) sont déterminés par les méthodes normalisées et ont été suivis pendant 3 ans (2003 à 2005).

Les concentrations de métaux ont été mesurées dans les sédiments à proximité de la décharge ainsi que dans le compost obtenu par compostage de déchets prélevés sur la décharge.

Tableau I : Variations des paramètres physico-chimiques du lixiviat

\begin{tabular}{|c|c|c|c|c|}
\hline & \multicolumn{2}{|c|}{$\begin{array}{c}\mathrm{P} 1, \mathrm{P} 2, \mathrm{P} 3, \mathrm{P} 4, \mathrm{P} 6, \mathrm{P} 7, \mathrm{P} 8 \\
\text { et P9 }\end{array}$} & \multicolumn{2}{|c|}{$\begin{array}{c}\text { P5 : point bas du site (en aval de la } \\
\text { décharge) }\end{array}$} \\
\hline & Intervalle de fléctuation & Moyenne & Intervalle de fléctuation & moyenne \\
\hline Turbidité (NTU) & $7-285$ & 104 & $78-280$ & 168 \\
\hline $\mathrm{pH}$ & $7.2-8.4$ & 7.9 & $7.6-7.9$ & 7.76 \\
\hline$\chi(\mathrm{ms} / \mathrm{cm})$ & $14.14-73.3$ & 42.4 & $23.06-52$ & 38.03 \\
\hline $\mathrm{O}_{2}(\mathrm{mg} / \mathrm{l})$ & $0-1.43$ & 0.21 & $0-0.1$ & 0.06 \\
\hline $\mathrm{DCO}(\mathrm{mg} / \mathrm{l})$ & $958-10100$ & 4260 & $2423-4644$ & 3385 \\
\hline $\mathrm{NO}_{3}(\mathrm{ppm})$ & $10.21-80.7$ & 35.64 & $9.2-11.5$ & 10.33 \\
\hline $\mathrm{NO}_{2}^{-}(\mathrm{ppm})$ & $0.4-28.3$ & 8.65 & $1.02-2.3$ & 1.66 \\
\hline NTK $(\mathrm{mg} / \mathrm{l})$ & $1050-3830$ & 2228 & $1163-1401$ & 1271 \\
\hline
\end{tabular}

Tableau 2 : Concentrations des éléments métalliques dans les lixiviats

\begin{tabular}{|l|l|l|l|l|l|l|l|l|}
\cline { 2 - 8 } \multicolumn{1}{c|}{} & $\begin{array}{l}\mathrm{Cr} \\
\mathrm{mg} / 1\end{array}$ & $\begin{array}{l}\mathrm{Cu} \\
\mathrm{mg} / 1\end{array}$ & $\begin{array}{l}\mathrm{Fe} \\
\mathrm{mg} / 1\end{array}$ & $\begin{array}{l}\mathrm{Pb} \\
\mathrm{mg} / \mathrm{l}\end{array}$ & $\begin{array}{l}\mathrm{Mn} \\
\mathrm{mg} / 1\end{array}$ & $\begin{array}{l}\mathrm{Ni} \\
\mathrm{mg} / 1\end{array}$ & $\begin{array}{l}\mathrm{Zn} \\
\mathrm{mg} / 1\end{array}$ & $\begin{array}{l}\mathrm{Sr} \\
\mathrm{mg} / 1\end{array}$ \\
\hline P1 & $1,5-3,7$ & $0,8-1,1$ & $5-75$ & 1,4 & $0,3-8,3$ & 0,5 & $1,5-2,8$ & $3,6-8,2$ \\
\hline P2 & $3,5-8,2$ & $0,3-4,3$ & $10-23$ & - & $0,3-1,8$ & - & $1,3-5,3$ & $1,6-9,4$ \\
\hline P3 & $3,5-5,7$ & $0,3-0,6$ & $7-20$ & - & $0,3-0,7$ & - & $1,3-5,8$ & $1,8-3$ \\
\hline P4 & $3,2-5,2$ & $0,3-0,5$ & $16-22$ & 2,1 & $1-1,1$ & - & $1,9-3,7$ & $3,4-5,4$ \\
\hline P5 & $6,2-9,0$ & $0,3-0,7$ & $18-40$ & - & $0,4-1$ & - & $1,4-3,7$ & $1,8-2,2$ \\
\hline P6 & $6,7-8,5$ & $0,3-0,6$ & $13-42$ & - & $0,4-0,8$ & - & $1,5-5,1$ & $1,2-2$ \\
\hline P7 & $5,7-8,2$ & $0,3-0,4$ & $9-33$ & 1,6 & $0,3-0,9$ & 0,6 & $2,2-6,3$ & $1,8-2,2$ \\
\hline P8 & $1,0-1,7$ & $0,3-0,4$ & $11-45$ & - & $0,3-1,6$ & - & $2,2-2,9$ & $1,6-7,8$ \\
\hline P9 & $3,6-4,2$ & $0,3-0,4$ & $5-8$ & - & $0,2-2,1$ & 0,6 & $1,1-2,2$ & $1,2-1,6$ \\
\hline
\end{tabular}

Figure I : Répartition des points de prélèvement de lixiviat

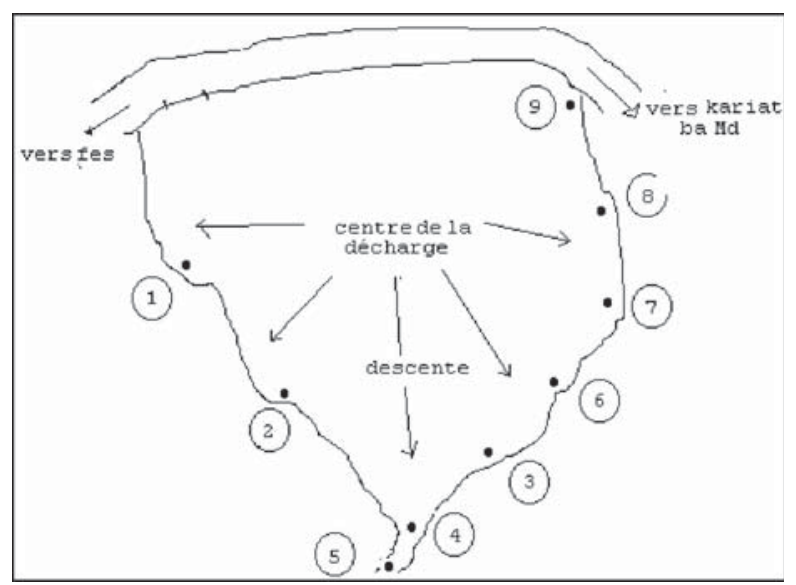

\section{Résultats}

Les paramètres physico-chimiques des lixiviats ont été suivis de 2003 à 2005. Ils sont rapportés dans le tableau I. Ces résultats révèlent de grandes variations de la composition chimique des lixiviats au cours du temps.

Les concentrations de certains éléments métalliques ( $\mathrm{Cu}$, $\mathrm{Zn}$, Fe, Cr, Ni, Pb, Sb, Sn) dans le lixiviat sont rapportées dans le tableau 2. Chaque valeur est la moyenne obtenue après 4 campagnes de prélèvement.

Les résultats d'analyse des éléments métalliques dans les sédiments prélevés aux différents points de la décharge sont rapportés dans le tableau 3.

\section{Analyse des métaux lourds dans le compost}

En outre, nous avons analysé les éléments métalliques (As, Cd, Cr, Cu, Fe, Hg, Ni, Pb et Zn) dans le compost maturé, obtenu à partir de déchets de la décharge, afin de d'avoir une idée sur le devenir des éléments métalliques durant le compostage. Les résultats sont rapportés dans le tableau 4.

\section{Discussion}

Les paramètres physico-chimiques des lixiviats déterminés entre les années 2000 et 2005 sont très variables.

La variation du $\mathrm{pH}$ pour les différents points de prélèvement est présentée sur la figure 2. Le $\mathrm{pH}$ varie entre 7 et 8,5 pour les différents points de prélèvements. La teneur en DCO varie d'un point de prélèvement à l'autre et avec la date de prélèvement (figure 3). Les valeurs de la DCO obtenues durant cette étude varient entre 958 et $10100 \mathrm{mg} / \mathrm{l}(19,2 \mathrm{~kg} / \mathrm{j}$ et $202 \mathrm{~kg} / \mathrm{j})$, valeurs inférieurs à 
Tableau 3 : Analyse des éléments métalliques accumulés dans les sédiments

\begin{tabular}{|l|l|l|l|l|l|l|l|l|l|}
\hline & $\begin{array}{l}\text { As } \\
\mu \mathrm{g} / \mathrm{g}\end{array}$ & $\begin{array}{l}\mathrm{Cd} \\
\mu \mathrm{g} / \mathrm{g}\end{array}$ & $\begin{array}{l}\mathrm{Cr} \\
\mu \mathrm{g} / \mathrm{g}\end{array}$ & $\begin{array}{l}\mathrm{Cu} \\
\mu \mathrm{g} / \mathrm{g}\end{array}$ & $\begin{array}{l}\mathrm{Fe} \\
\mathrm{mg} / \mathrm{g}\end{array}$ & $\begin{array}{l}\mathrm{Hg} \\
\mu \mathrm{g} / \mathrm{g}\end{array}$ & $\begin{array}{l}\mathrm{Ni} \\
\mu \mathrm{g} / \mathrm{g}\end{array}$ & $\begin{array}{l}\mathrm{Pb} \\
\mu \mathrm{g} / \mathrm{g}\end{array}$ & $\begin{array}{l}\mathrm{Zn} \\
\mu \mathrm{g} / \mathrm{g}\end{array}$ \\
\hline P1 & 134,5 & 3,94 & 1141,8 & 297,5 & 203,76 & 2,55 & 226,8 & 222 & 934,8 \\
\hline P2 & 59,6 & 2,50 & 530,7 & 493,2 & 131,37 & 1,80 & 197,5 & 170 & 608,6 \\
\hline P3 & 89,5 & 1,70 & 848,5 & 1382,7 & 212,19 & 3,60 & 298,6 & 760 & 905,1 \\
\hline P4 & 59,4 & 1,85 & 768,1 & 211,9 & 267,75 & 4,72 & 310,1 & 153,5 & 1025,1 \\
\hline P5 & 34,7 & 2,50 & 663,8 & 261,8 & 112,16 & 1,84 & 175,1 & 124,5 & 466,1 \\
\hline P6 & 60,9 & 2,30 & 1255,6 & 224,8 & 172,82 & 2,22 & 247,8 & 188,8 & 694,4 \\
\hline P7 & 41,6 & 1,56 & 1098,7 & 93,1 & 181,14 & 3,35 & 237,1 & 138,6 & 711,5 \\
\hline P8 & 55,4 & 0,87 & 1020,3 & 84,4 & 193,27 & 3,33 & 262,3 & 101,4 & 549,7 \\
\hline P9 & 49,9 & 1,20 & 531,9 & 261,1 & 228,76 & 3,33 & 305,9 & 180,7 & 590,7 \\
\hline
\end{tabular}

Tableau 4 : Concentrations en éléments métalliques dans le compost

\begin{tabular}{|c|c|c|c|c|c|c|c|c|}
\hline $\begin{array}{c}\mathrm{As} \\
\mu \mathrm{g} / \mathrm{g}\end{array}$ & $\begin{array}{c}\mathrm{Cd} \\
\mu \mathrm{g} / \mathrm{g}\end{array}$ & $\begin{array}{c}\mathrm{Cr} \\
\mu \mathrm{g} / \mathrm{g}\end{array}$ & $\begin{array}{c}\mathrm{Cu} \\
\mu \mathrm{g} / \mathrm{g}\end{array}$ & $\begin{array}{c}\mathrm{Fe} \\
\mathrm{mg} / \mathrm{g}\end{array}$ & $\begin{array}{c}\mathrm{Hg} \\
\mu \mathrm{g} / \mathrm{g}\end{array}$ & $\begin{array}{c}\mathrm{Ni} \\
\mu \mathrm{g} / \mathrm{g}\end{array}$ & $\begin{array}{c}\mathrm{Pb} \\
\mu \mathrm{g} / \mathrm{g}\end{array}$ & $\begin{array}{c}\mathrm{Zn} \\
\mu \mathrm{g} / \mathrm{g}\end{array}$ \\
\hline 48,1 & 0,73 & 480,8 & 71,6 & 250,72 & 3,25 & 305,4 & 111,1 & 1320,5 \\
\hline
\end{tabular}

celles obtenues par exemple à la décharge de la ville de Mohammedia (Chtioui et al., 2005).

Le débit du lixiviat s'écoulant en bas de la décharge, vers l'oued Fès est d'environ $20 \mathrm{~m}^{3} / \mathrm{j}$. La charge polluante ainsi produite par les rejets de lixiviats varie au cours du temps et peut atteindre $202 \mathrm{~kg} / \mathrm{j}$. Le débit mesuré à la décharge de Fès est supérieur à celui mesuré à la décharge de la ville de Mohammedia (Chtioui et al., 2005) mais reste inférieur à celui mesuré à la décharge de Tanger : 86,4 m³/j (Ariane 2004).

Figure 2 : Variation du $\mathrm{pH}$ du lixiviat au cours du temps pour différents points de prélèvement

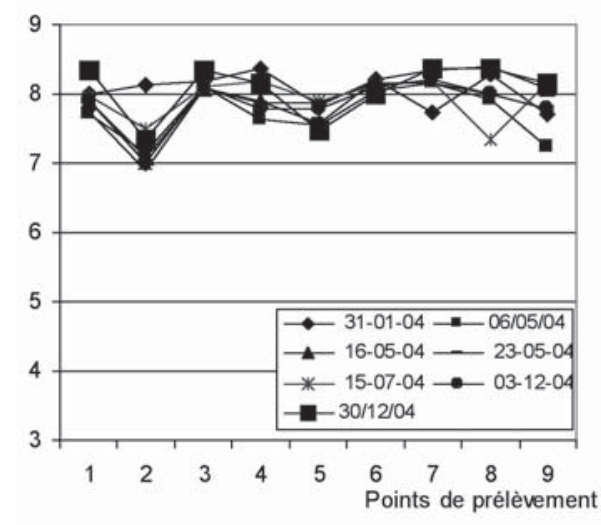

Figure 3 : Variation du la DCO du lixiviat au cours du temps pour différents points de prélèvement

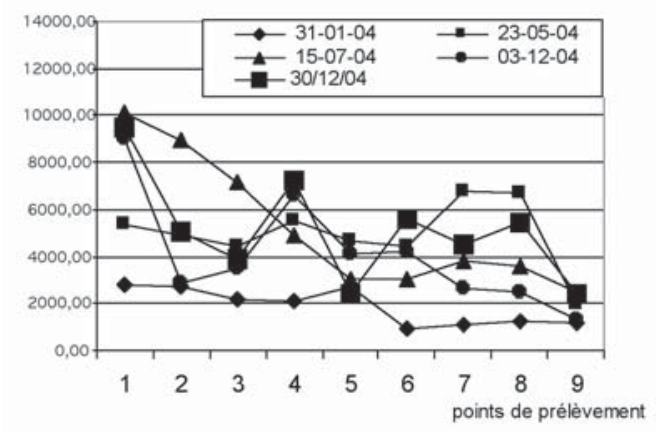

La concentration en NTK détectée dans le lixiviat de la décharge de la ville de Fès varie d'un point à l'autre et au cours temps (figure 4). La teneur en NTK peut atteindre 4000 mg/l avec une moyenne de 2200 mg/l, ce qui est comparable aux résultats obtenus sur le lixiviat de la décharge de la ville de Mohammedia : $3000 \mathrm{mg} / \mathrm{l}$ (Chtioui et al. 2005). Il est à noter que la concentration en nitrate peut atteindre $80 \mathrm{mg} / \mathrm{l}$ à certains points de prélèvements, ce qui dépasse les normes de rejets déversés dans le milieu récepteur.

Figure 4 : Variation du NTK du lixiviat au cours du temps pour différents points de prélèvement

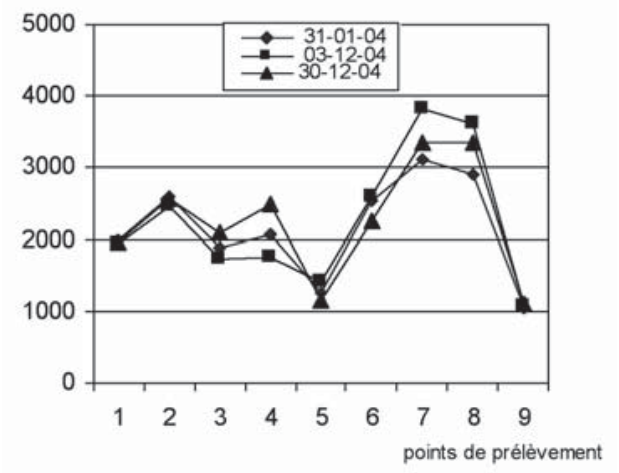

Par ailleurs, on observe que la teneur en chrome du lixiviat varie entre I et $9 \mathrm{mg} / \mathrm{l}$ et reste supérieure à la concentration $(5 \mathrm{mg} / \mathrm{l})$ détectée dans le lixiviat de la décharge de Mohammedia (Chtioui et al.2005) et à celle mesurée à la décharge d'Akreuch (Wilaya Rabat-Salé) (ONEP 1998) (35-120 $\mu \mathrm{g} / \mathrm{l})$. Ceci est dû au fait que plusieurs unités industrielles de tannerie sont installées à Fès. Cette présence de chrome peut provenir d'autres types de déchets collectés avec les ordures ménagères comme les papiers cartons et le bois (Cr total $=25 \mathrm{mg} / \mathrm{g}$ de papier et $80 \mathrm{mg} / \mathrm{g}$ de bois) (El M'ssari, 1993). II est à noter que la décharge de la ville de Fès est une décharge « ouverte ». Elle reçoit toutes sortes de déchets urbains et industriels en l'absence d'une réglementation permettant d'aboutir à une gestion rigoureuse d'une décharge. 
L'analyse des éléments métalliques dans les sédiments a montré que la teneur de ces éléments varie d'un point de prélèvement à l'autre. La concentration en Cr varie entre 480 et I $250 \mu \mathrm{g} / \mathrm{g}$ ce qui est en relation avec la concentration du Cr dans le lixiviat qui peut atteindre $9 \mathrm{mg} / \mathrm{l}$. Ceci témoigne d'une forte pollution prévenant des unités industrielles de tannerie qui rejettent une quantité importante de déchets sans aucun traitement préalable en absence d'une réglementation. La concentration en $\mathrm{Pb}$ atteint $760 \mu \mathrm{g} / \mathrm{g}$ pour le point P3. Cela est en relation avec les huiles de vidange rejetées à la décharge sans savoir que les rejets peuvent être valorisés. En outre, la concentration en $\mathrm{Hg}$ accumulée par les sédiments reste très importante et dépasse 4,5 $\mu \mathrm{g} / \mathrm{g}(\mathrm{P} 4)$ avec un minimum de 1,80 $\mu \mathrm{g} / \mathrm{g}(\mathrm{P} 2)$. Ceci montre que les déchets solides rejetés à la décharge sont riches en matière contenant des piles ou autres types de déchets riches en $\mathrm{Hg}$. En ce qui concerne le $\mathrm{Cd}$, la concentration accumulée par les boues varie entre 0,7 et 3,94 $\mu \mathrm{g} / \mathrm{g}$, ce qui témoigne d'une forte pollution des rejets de lixiviat provenant de la fermentation des déchets solides reçus à la décharge de la ville de Fès.

Le compost contient également des concentrations importantes en $\mathrm{Cr}$ (480,8 mg/g), ainsi qu'en $\mathrm{Zn}$ ( $1320,5 \mathrm{mg} / \mathrm{g}$ ). Ceci est en relation avec la concentration du Cr analysée dans le lixiviat. La concentration en Cr dans le compost peut empêcher l'utilisation du compost comme fertilisant puisque ce métal peut facilement s'accumuler sur les plantes. De même, la concentration du $\mathrm{Pb}$ est élevée et varie autour de I I I $\mu \mathrm{g} / \mathrm{g}$ de sédiment (ceci est en relation avec les déchets tels que les huiles de vidange).

\section{Conclusion}

Les résultats de cette étude ont mis en évidence la pollution générée par le lixiviat de la décharge de la ville de Fès. Les résultats ainsi obtenus marquent une forte teneur en DCO (entre 958 et 10100 mg/l) dont la matière polluante est difficilement décantable et non biodégradable. La concentration en NTK varie entre I 050 et 3830 $\mathrm{mg} / \mathrm{l}$ tandis que la teneur en nitrates dépasse $80 \mathrm{mg} / \mathrm{l}$.

La présence des éléments métalliques détectée dans les lixiviats montre que les ordures ménagères sont mélangées avec les rejets industriels. La teneur en chrome détectée dans les rejets de lixiviats varie avec les points de prélèvement et au cours du temps et peut atteindre $9 \mathrm{mg} / \mathrm{l}$, dépassant ainsi les normes de rejets, ce qui constitue un vrai danger pour l'environnement et la santé publique. Les teneurs des éléments métalliques de la plupart des éléments analysés dans le lixiviat et dans ceux accumulés par les sédiments restent très importantes et dépassent la réglementation recommandée dans les pays en voie de développement. L'analyse des éléments métalliques tels que $\mathrm{Cr}$, Fe, $\mathrm{Ni}, \mathrm{Pb}$, $\mathrm{Zn}, \mathrm{Sn}, \mathrm{Sb}$ et $\mathrm{Cu}$ dans les eaux de lixiviats et dans les sédiments prélevés dans les neuf points choisis pour l'étude de la décharge de la ville de Fès a montré des teneurs importantes en ces éléments. La concentration en Cr dans le cas du sédiment varie entre 530 et I $255 \mu \mathrm{g} / \mathrm{g}$, tandis que le $\mathrm{Pb}$ varie entre 100 et $760 \mu \mathrm{g} / \mathrm{g}$. Ceci témoigne d'une pollution par plusieurs types de rejets industriels riches en métaux, notamment le $\mathrm{Cr}$ dont la présence est causée par les déchets solides rejetés par les unités industrielles (tanneries) de la ville sans aucun traitement préalable en absence de réglementation.

\section{Références}

Ariane B., 2003. Endommagement de l'environnement par l'exploitation de la décharge publique de Tanger, Maroc, Coopération Maroc-Allemagne : rapport Programme Gestion Protection de l'Environnement (PGPE).

Chtioui H., Souabi S., Aboulhassan M.A., Zakarya D., 2005. Contribution à l'évaluation de la pollution générée par les lixiviats de la décharge de la ville de Mohammedia et son impact sur l'environnement, 58, 283, Eau Industrie et Nuisances.

Crawford J.F., Smith PG., 1985. Landfill Technology. Butterworths, London.

El M'ssari N., 1993. Elaboration d'une méthodologie de caractérisation des ordures ménagères et du compost de l'usine de Salé. Thèse DES. Faculté des sciences, Meknès.

Elkhamlichi M.A., Lakranbi S., Kabbaj M., Jabri E., Kouhen M., 1997. Etude d'impact de la décharge d'Akrach sur la qualité des ressources en eau. Revue marocaine de génie civil. 68 :17-30.

Fekri A., Wahbi M., Ben Bouzian A., Souabi S., Marrakchi M., 2004. Etat de la qualité des eaux souterraines en aval de la décharge de Mediouna (Casablanca, Maroc). The First international symposium on the management of liquid and solid residus (MALISORE). Mohammedia, Maroc.

Kerrbachi R., Bekacemi. 1994. Caractérisation et évolution des lixiviats de la décharge de Oued Smar à Alger.TMS. II.

ONEP (Office national de l'eau potable), 1998. Caractérisation physico-chimique et bactériologique du lixiviat de la décharge d'Akreuch. Rapport sur la décharge d'Akreuch, Rabat, Maroc.

DÉCHETS SCIENCES \& TECHNIQUES, REVUE FRANCOPHONE D'ÉCOLOGIE INDUSTRIELLE SAP - 9 rue de l'Arbre Sec - 6928I LYON CEDEX 0I- Mèle : olivier.guichardaz@pro-environnement.com

Service abonnement : SAP/DPE - Service abonnement - 9 rue de l'Arbre Sec - 6928I LYON CEDEX 0 I

Tél. : 0472982669 - Fax : 0472982680

$N^{\circ}$ de commission paritaire : 0307 T 88295 - N ISSN : 0753-3454. Dépôt légal : À parution - Imprimerie Louis Jean/Gap - Photocomposition SAP

Principaux associés : DPE 